\title{
CFD Analysis for Optimum Thermal Design of a Carbon Nanotube Based Micro-channel Heatsink
}

\author{
Farhad Ismail*, A. I. Rashid, and M. Mahbub \\ Department of Mechanical Engineering, Bangladesh University of Engineering \& Technology, \\ Bangladesh \\ E-mail: farhadananda.128@gmail.com*
}

\begin{abstract}
Carbon nanotube (CNT) is considered as an ideal material for thermal management in electronic packaging because of its extraordinary high thermal conductivity. A series of 2D and 3D CFD simulations have been carried out for CNT based micro-channel cooling architectures based on one and two dimensional fin array in this paper using COMSOL 4.0a software. Micro-channels are generally regarded as an effective method for the heat transfer in electronic products. The influence of various fluids, micro-fin structures, micro-fin array, fluid velocity and heating powers on cooling effects have been simulated and compared in this study. Steady-state thermal stress analyses for the forced convection heat transfer have also been performed to determine maximum allowable stress and deflections for the different types of cooling assembly.
\end{abstract}

Keywords: CNT, micro-channel, CFD, cooling effects, thermal stress.

ENGINEERING JOURNAL Volume 15 Issue 4

Received 9 July 2011

Accepted 31 August 2011

Published 1 October 2011

Online at http://www.ej.eng.chula.ac.th/eng/

DOI:10.4186/ej.2011.15.4.11 


\section{Introduction}

Carbon nanotubes (CNTs) were discovered in 1991. They are allotropes of carbon with a nanostructure that have a length-to-diameter ratio greater than 1000000. Figure 1 shows the structure of single-walled carbon nanotubes (SWNTs). A CNT is formed when the two-dimensional sheet of graphene is rolled into a seamless cylinder. CNTs can range from $\sim 1 \mathrm{~nm}$ to $\sim 100 \mathrm{~nm}$ in diameter and have lengths in the micrometer range. Based on the chemical arrangement of carbon atoms, a discrete number of unique CNTs can be formed. CNTs are made from cylindrical carbon molecules which are very special in thermal, electrical and mechanical properties. A thermal conductivity up to $6600 \mathrm{~W} / \mathrm{m} \cdot \mathrm{K}$ has been reported [1]. Copper, silver and gold, which are some of the best known thermally conductive materials, have thermal conductivities of $400 \mathrm{~W} / \mathrm{m} \cdot \mathrm{K}, 430 \mathrm{~W} / \mathrm{m} \cdot \mathrm{K}$ and $320 \mathrm{~W} / \mathrm{m} \cdot \mathrm{K}$ at room temperature respectively. As a new material, CNTs are attracting more and more attention, and they are potentially very useful in nanotechnology, electronics, optics and aeronautics. CNTs can be manufactured by Chemical Vapor Deposition (CVD) at suitable temperature. During the CVD process, process gas (such as ammonia, nitrogen, hydrogen, etc.) and carbon-containing gas (such as acetylene, ethylene, ethanol, methane, etc.) are demanded as reactors, and catalyst particles such as nickel, cobalt and iron are also needed. Therefore, to promote a good thermal contact on the interfaces between the multi-walled CNTs and their growth substrate, adhesion layers consisting titanium, molybdenum or chromium were often deposited onto the substrates before fabrication [2-4]. Other studies showed that the multi-walled CNT free ends' interface had significant higher resistance compared to that at the multi-walled CNT growth substrate interface; and this problem could be solved by using a thin layer of indium to weld the multiwalled CNT ends to opposing substrate. CNT grows at high temperatures of about $750^{\circ} \mathrm{C}$. In order to prevent the chip from being damaged, the nanotubes could be grown on silicon substrates and there after transferred onto the chip. The bond between the CNTs and the chip is crucial.

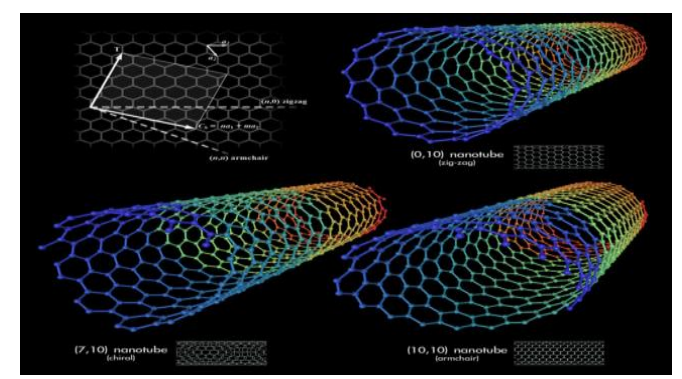

Fig. 1. Single-walled carbon nanotubes [3].

\section{Micro-channel Cooler}

Micro-channel cooler is a very promising approach to meet the requirements of microelectronics package cooling. A lot of investigations about micro-channels have been undertaken in the past years. A silicon micro-channel can remove $790 \mathrm{~W} / \mathrm{cm}^{2}$ heat with a temperature rise of 71 degrees between the substrate and the coolant. The width and height of the silicon channel are $50 \mu \mathrm{m}$ and $302 \mu \mathrm{m}$ separately. However, as the trends in the electronics industry moves towards higher packaging density, the highpressure drop problem limits the performance of traditional silicon heat sink. Replacing the silicon fins with nanotube fins to enhance the thermal exchange rate between cooling liquid and substrate is one way to overcome this problem. Growing aligned nanotubes on the whole substrate is another one. A typical micro-channel cooler includes cover on the top; silicon substrate at bottom with the microchannels is showed in Fig. 2. Channels are etched and covered by plexiglass on the top and ultimately formed a complete structure [2]. The basic principle of micro-channel heat is that bottom is in touch with the heat and fluid flows through the entrance to the export to take away heat. In practice, with increasing heat, when the micro-cooler maximum temperature exceeds the fluid's temperature, convection heat is generated between wall and fluid until the heat balance is stabilized and micro-cooler works into the stable working condition. Replacing the silicon fins with nanotube fins or growing aligned nanotubes on the whole substrate to enhance the thermal exchange rate between cooling liquid and substrate is one way to overcome this problem. 


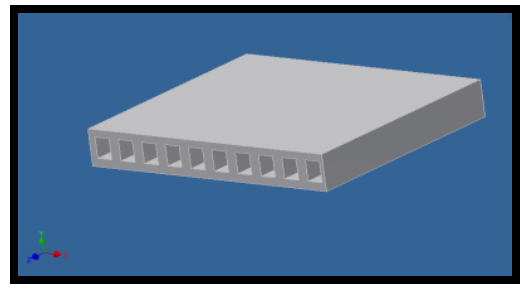

Fig. 2. Micro-channel cooler.

\section{CNT Based Micro-channel Cooler}

CNT as fin material was introduced by Liu and his research group in 2004. A bare silicon chip was chosen as the substrate. After CVD synthesis, CNTs array [5-7] was grown from the catalyst as cooling fins (Fig. 3). Finally, a lid was bonded to seal the CNTs and form the micro-channels. Figure 3 shows the flow chart. Figure 4 shows the SEM pictures of the one-dimensional and two-dimensional CNTs arrays respectively.

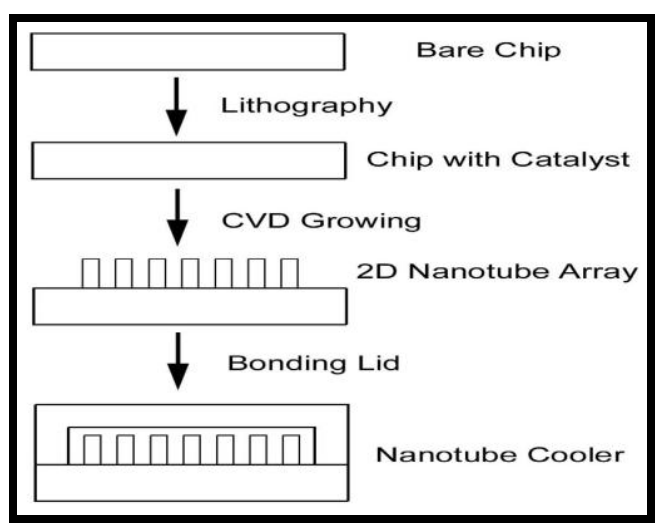

Fig. 3. Process to manufacture CNT based micro-channel cooler.

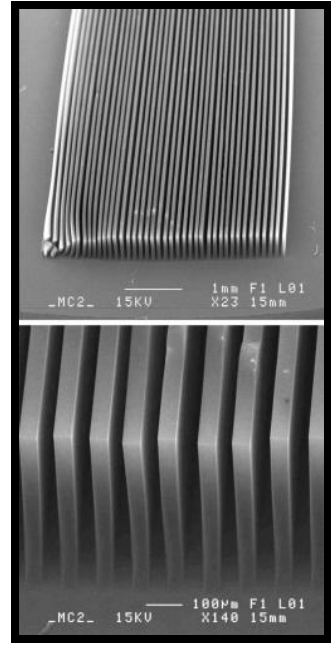

(a)

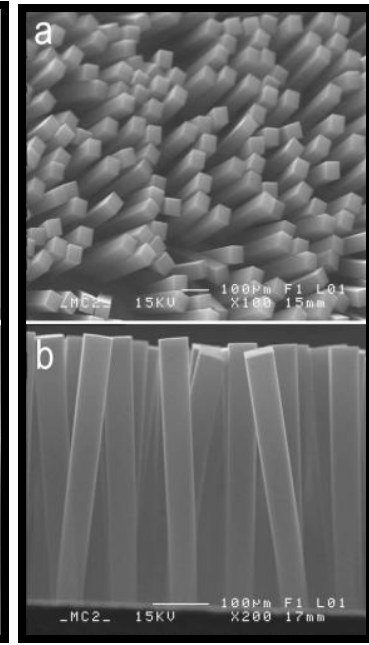

(b)

Fig. 4. SEM picture of CNT with one and two dimensional array: (a) one dimensional array; (b) two dimensional nano-tube array [5]. 


\section{CFD Simulation Model}

\subsection{Theoretical Analysis}

A coolant flows through a micro channel heat sink described before takes away heat from heat component attached below (constant heat flux). The top face is made of insulated material (such as glass) and the bottom material is silicon. The heat transfer contains two parts: conduction in the solid and convection between the solid and coolants. By continuities of temperature and heat flux, the solid region and fluid region are coupled. Some simplifying assumptions are considered as follows:

(1) Laminar flow;

(2) Incompressible flow;

(3) Hydro dynamically and thermally fully developed;

(4) No radiation of the wall;

(5) Negligible convection of air out of the cooling assembly;

(6) Constant solid and fluid properties.

\subsection{Simulation model and validation}

In this study (Fig. 5) two and three dimensional works were investigated. For this cases the energy conservation equation, which can theoretically predict the value of temperature rise between the inlet and outlet, was used for simulation validation in this work. Following the adiabatic boundary conditions in this simulation, the energy supplied by the chip should be equal to the heat removed by the coolant. $\mathrm{T}_{\text {in }}$ is inlet temperature of the channel $\left(20^{\circ} \mathrm{C}\right)$. A typical parallel plate heat sink is showed for the microfin. The CNT array was grown on a $10 \mathrm{~mm} \times 10 \mathrm{~mm}$ area. The thickness of the underneath silicon substrate (base height, $\mathrm{BH}$ ) is $50 \mu \mathrm{m}$. The channel is long enough so that fully developed flow can be attained. For 1D fin array arrangement from the following figure $\mathrm{L}=\mathrm{W}=10 \mathrm{~mm}$. Height $\mathrm{H}=0.65 \mathrm{~mm}$. Fin pitch (S) and fin thickness (t) are equal to $0.5 \mathrm{~mm}$ (Fig. 5-7). The surface area was kept constant for all the geometries. In Fig. 6, top view of different kind of fin geometry investigated here has been showed. These are 1D rectangular array, 2D rectangular array (square cross-section), 2D array with $45^{\circ}$ orientations with the flow and 2D rectangular array (circular cross-section) respectively. In Fig. 6, different types of cooling assembly is shown. The first one is one dimensional cooling array. All others are two dimensional micro-fin arrays. The third one is oriented $45^{\circ}$ with the velocity of fluid flow.

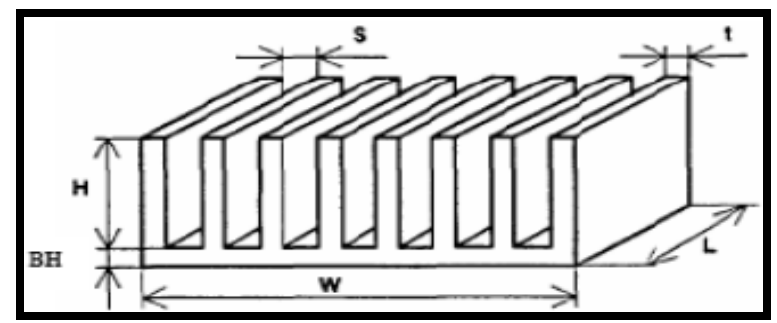

Fig . 5. Parallel plate heat sink showing all the dimensions.
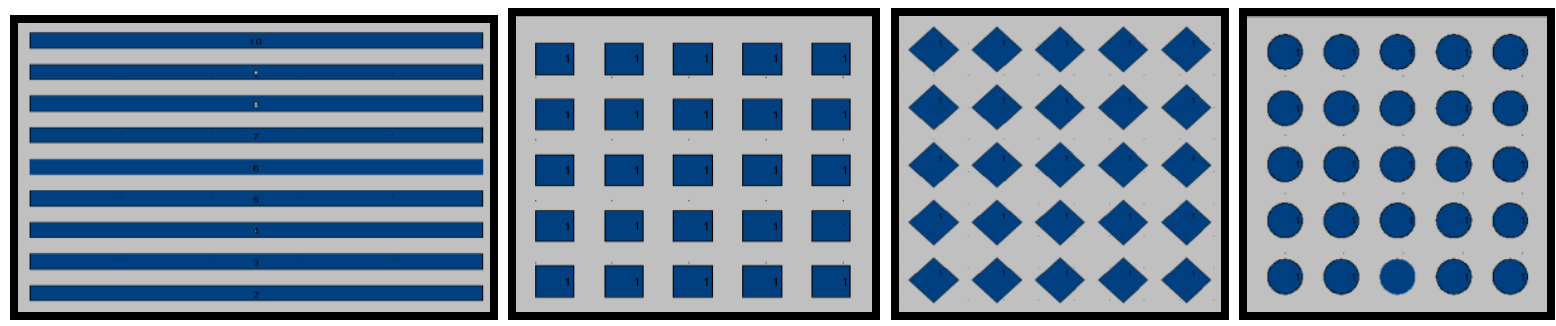

Fig. 6. Different orientation of CNT grown on silicon substrate. 


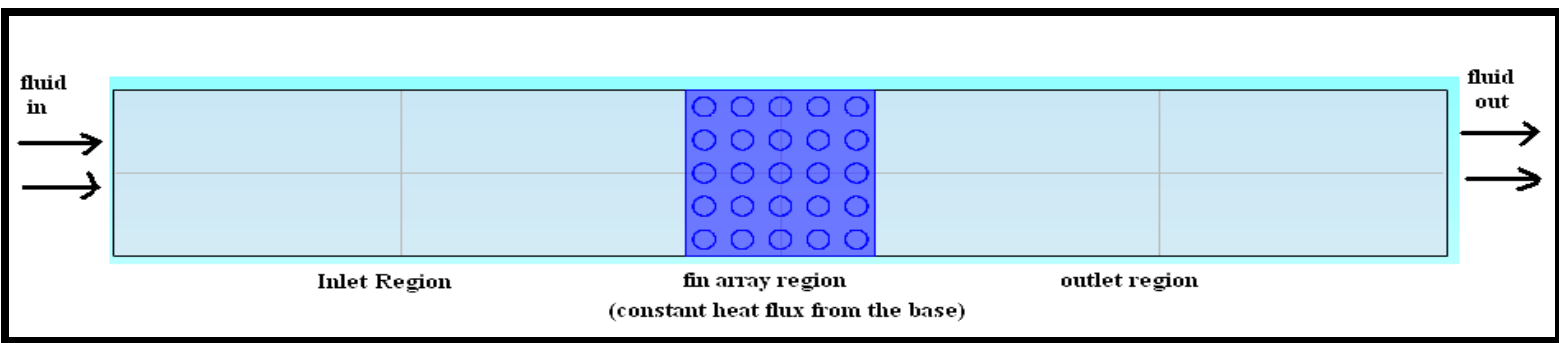

Fig. 7. CFD computational model (top view) for circular pin-fin heat sink.

The temperature of the cooling fluid at the end of the channel stayed almost constant. This can be explained by the conservation of energy. The energy equation of the coolant in the model is expressed as Eq. (1). If $\mathrm{L}_{\mathrm{w}}$ is width of cooler model then for $2 \mathrm{D}$ case:

$$
\frac{\int_{0}^{L_{W}} \rho_{f} c_{p, f} V(y)\left[T_{\text {out }}(y)-T_{\text {in }}\right] d y}{L_{W}}=W_{\text {chip }}
$$

The energy conservation equation, which can theoretically predict the value of temperature rise between the inlet and outlet, was used for simulation validation in this work. Following the adiabatic boundary conditions in this simulation, the energy supplied by the chip should be equal to the heat removed by the water Eq. (2):

$$
\rho_{f} V c_{p, f}\left(T_{\text {out }}-T_{\text {in }}\right)=W_{\text {chip }}
$$

where $\mathrm{V}$ is volume flow rate, $\mathrm{C}_{\mathrm{p}, \mathrm{f}}$ is specific heat of fluid at constant pressure, $\mathrm{T}_{\text {out }}$ is outlet fluid temperature, $\mathrm{T}_{\text {in }}$ is inlet fluid temperature $\left(20^{\circ} \mathrm{C}\right)$. The average water temperature $\left(\mathrm{T}_{\text {out }}\right)$ at the outlet of cooling assembly can be calculated by Eq. (3):

$$
T_{\text {out }}=\frac{\int_{0}^{L_{W}} T_{L_{3}}(y) d y}{L_{W}}
$$

where $\mathrm{L}_{\mathrm{w}}$ is width of cooler model, $\mathrm{L}_{3}$ is the overall length of the micro-channel.

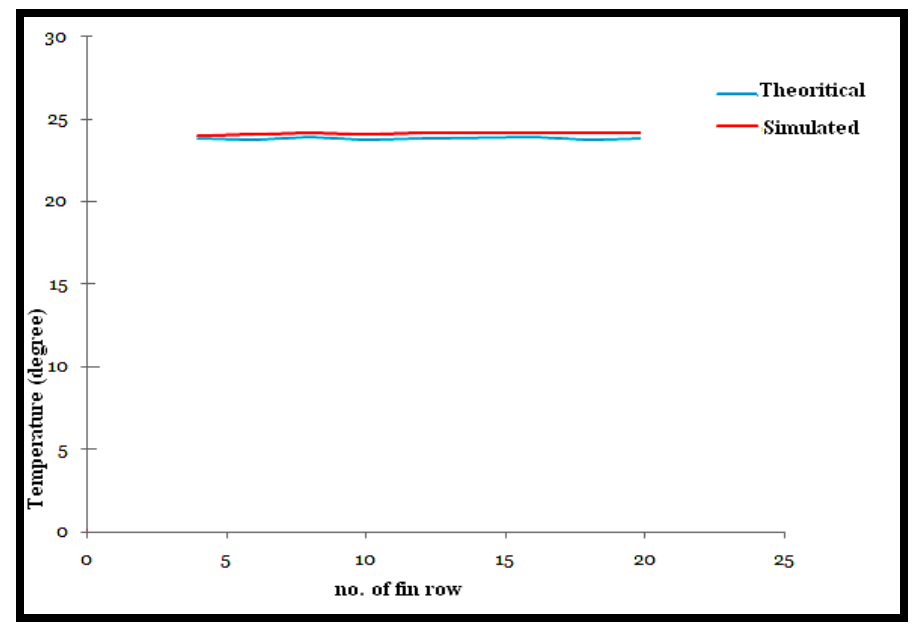

Fig. 8. Comparison between CFD simulation results and theoretical prediction of average water temperature at outlet for different number of fin row.

From Fig. 8, it is showed that there is a good agreement between the simulation results and theoretical calculation. Therefore it is indicated that the CFD simulation results in this work are reasonable and reliable. 


\section{Mesh Generation and mesh dependence test}

Conjugate heat transfer module is used to treat the solid and fluid as a unitary computational domain, and to solve the above governing equations simultaneously. The mesh in every channel should be fine enough (Fig. 9-10). Thermal conductivity of CNT is very high in z-direction (400 W/m-K) and low in $\mathrm{x}$ and y direction $(40 \mathrm{~W} / \mathrm{m}-\mathrm{K})$.

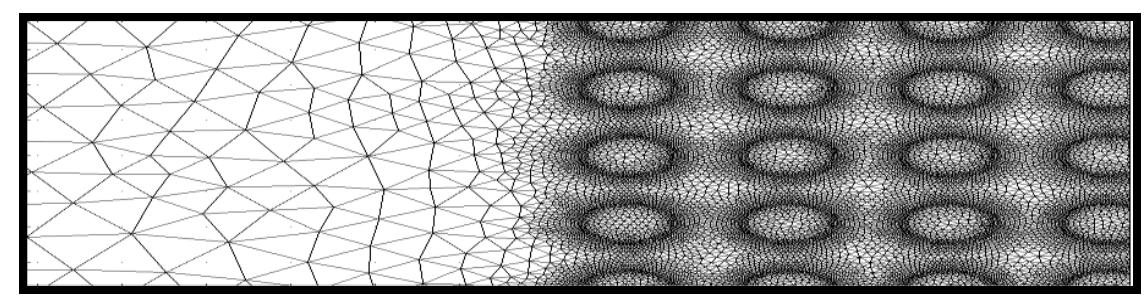

Fig. 9. 2D Mesh generation at fin assembly.
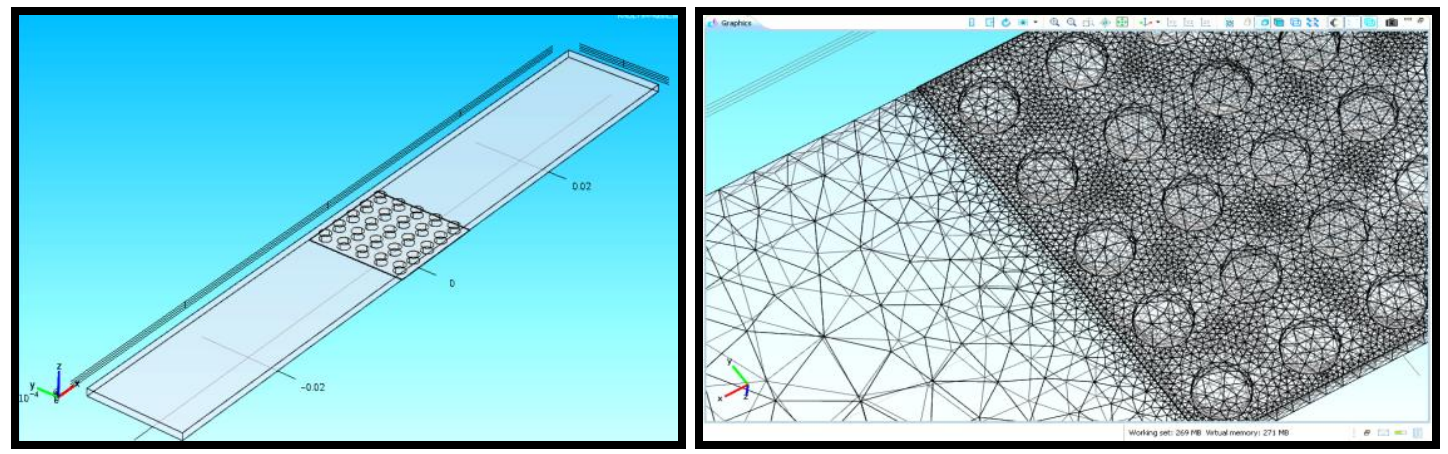

Fig. 10. 3D model and fine mesh generation at fin assembly.

Different kinds of mesh numbers had been carried out and compared with the maximum fin temperature (Fig. 11). The comparison graph is showed that there is not much difference in the results. So, the influence of amount of mesh can be neglected in the certain region.

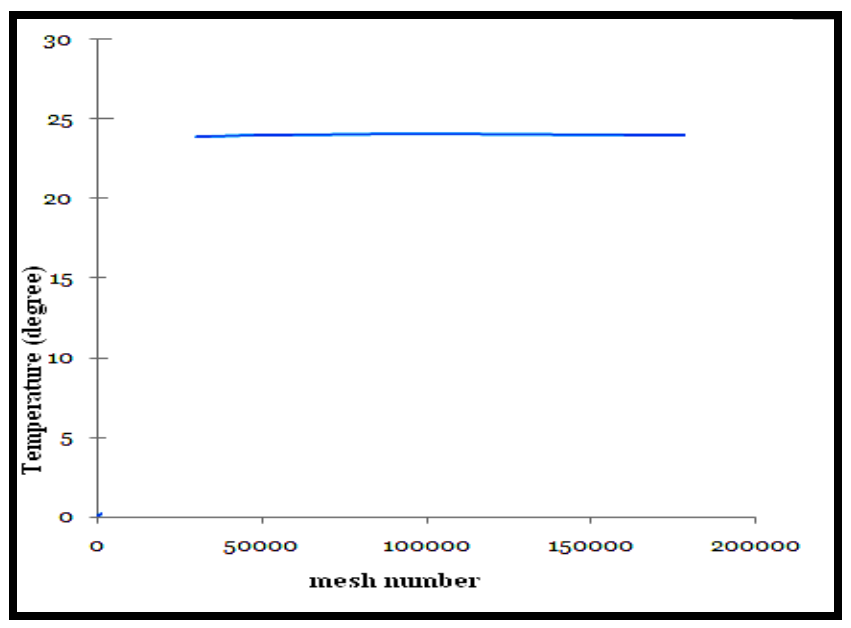

Fig.11. Mesh dependence test-maximum fin temperature vs. mesh number.

\section{Simulation Results And Analysis}

Here three dimensional models are simulated for different types of fin geometry; different fluid and fluid velocity. The inlet velocity was set $20^{\circ} \mathrm{C}$ and the heat flux was $15 \mathrm{~W} / \mathrm{cm}^{2}$ (Fig. 12-13). 


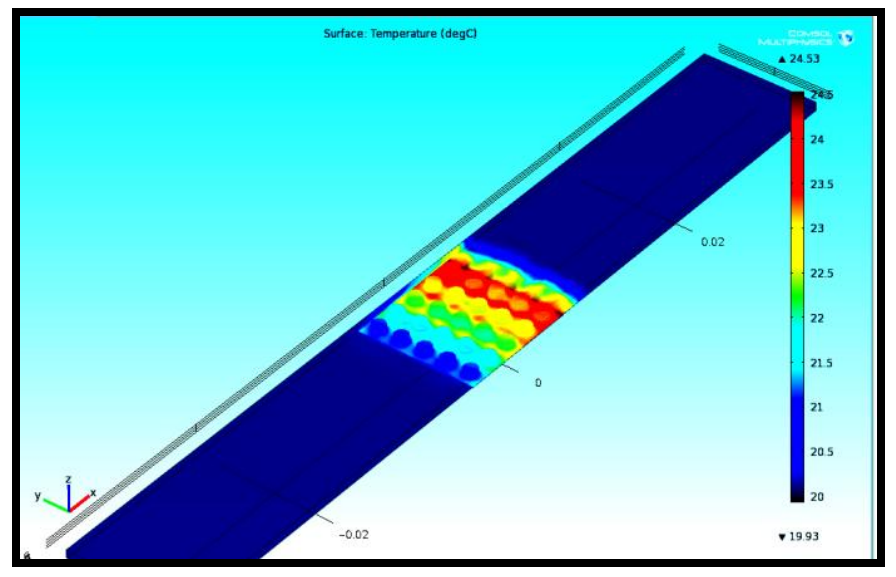

Fig. 12. 3D Temperature distribution for 2D circular fin array.

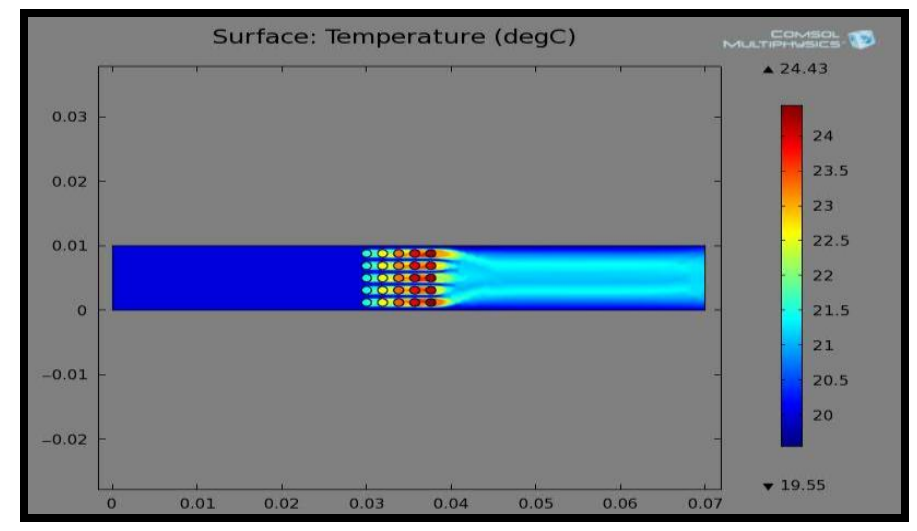

Fig. 13. 2D Temperature distribution for 2D circular fin array.

\subsection{Effect of fluid velocity}

The simulation result shows that maximum fin temperature decreases with increase of fluid velocity. Here four velocities $-0.1 \mathrm{~m} / \mathrm{s}, 0.25 \mathrm{~m} / \mathrm{s}, 0.5 \mathrm{~m} / \mathrm{s}$ and $0.75 \mathrm{~m} / \mathrm{s}$ are used (Fig. 14).

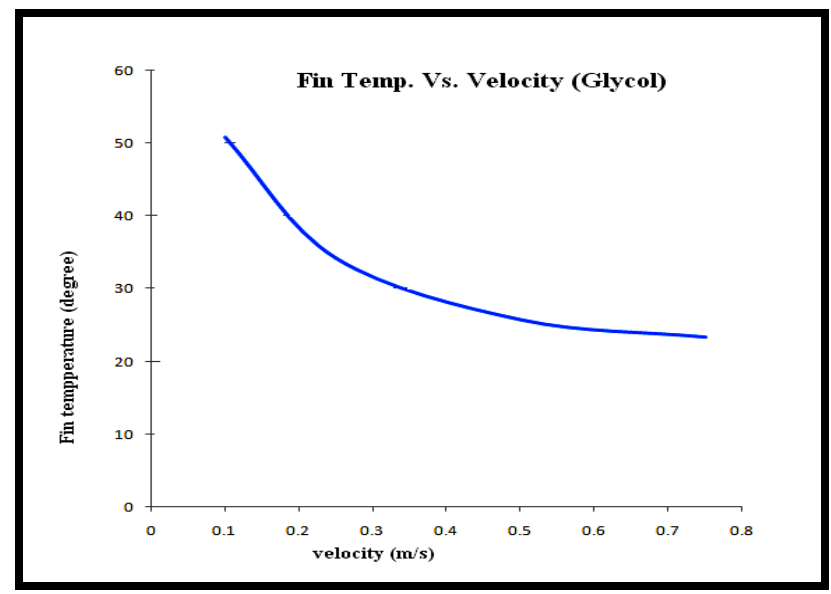

Fig. 14. Maximum fin temperature vs. velocity graph. 


\subsection{Effects of micro-fin Structure}

Four types of geometry are used to simulate the above model- 1D pattern array, 2D rectangular pattern array, 2D rectangular pattern with $45^{\circ}$ orientations, 2D circular fin array. The simulation results showed that 2D fin array is the efficient one among these fin geometries (Fig. 15).

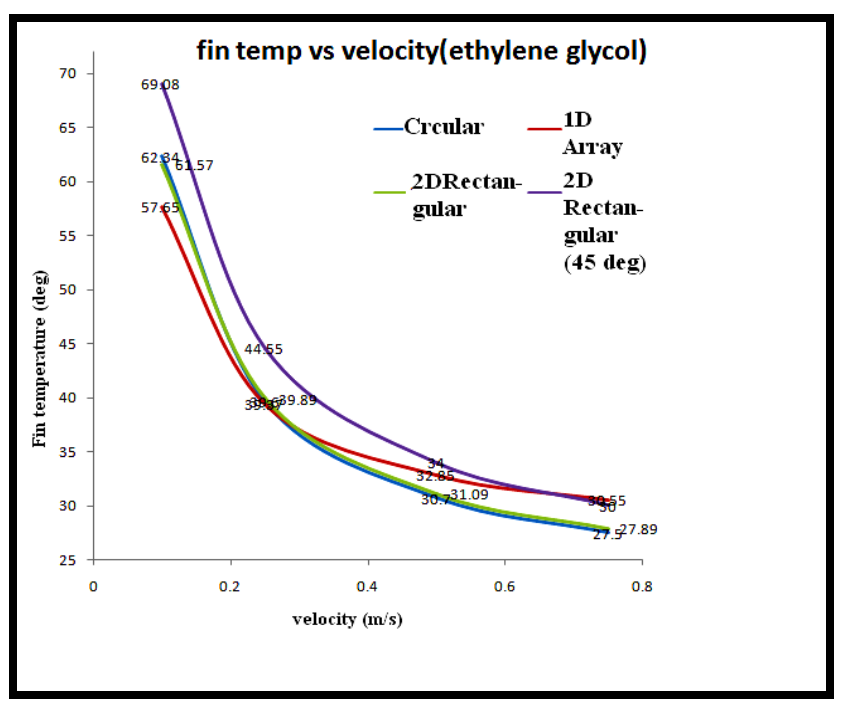

Fig. 15. Fin temperature vs. velocity for different fin geometry for glycol.

\subsection{Effects of fluid}

The heat transfer capability is dependent on the properties of working fluid, the most frequently used coolant in the micro channel heat sink is water. Better results may be possible with other fluids. So, one of the methods for changing heat transfer is to use the different working fluids. Here the model is simulated for four fluids-water, ethylene glycol, $30 \%$ ethylene glycol solution and ethanol (Fig. 16).

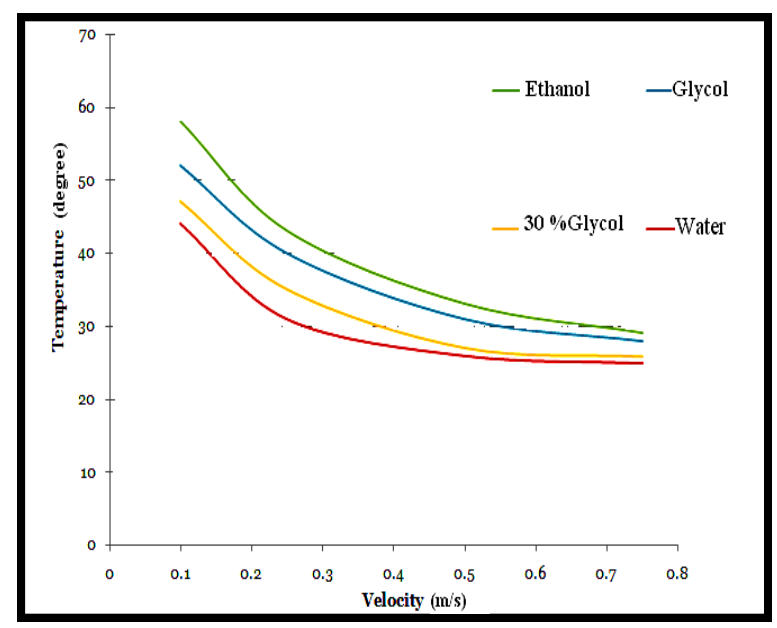

Fig. 16. Comparison graph for different fluid effectiveness.

\subsection{Effects of fin width}

From the 2D analysis it is investigated that the maximum fin temperature is decreased with increase of channel width up to a certain limit and then increased proportionally. Because, after crossing this limit the fluid can't be passed through the channels properly. The channel length is $70 \mathrm{~mm}$ and width is varied from $0.0005 \mathrm{~mm}$ to $0.0024 \mathrm{~mm}$ for the simulation. The analysis is done for $4 * 4$ two dimensional rectangular arrays (Fig. 17). 


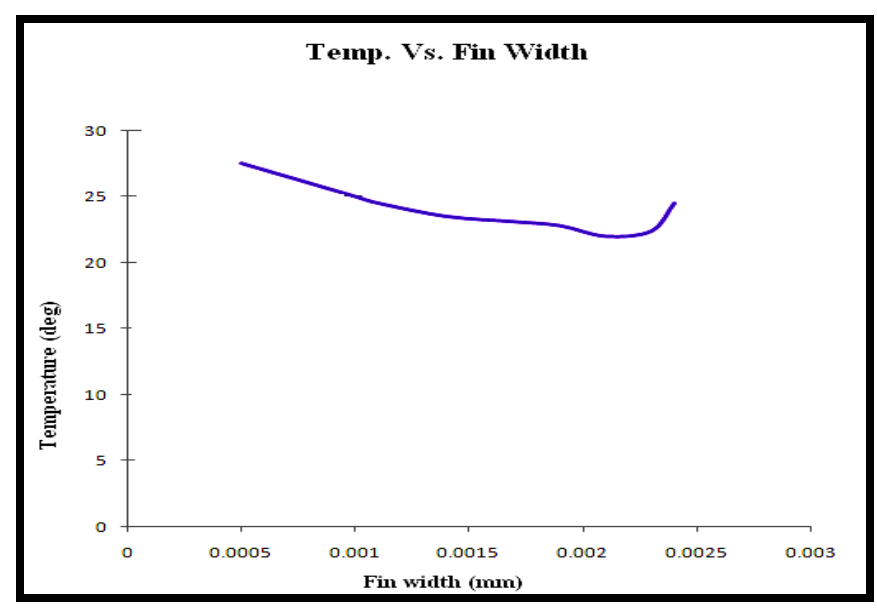

Fig. 17. Temperature vs. fin width.

\subsection{Effects of increasing the fin array}

It is investigated (2D CFD analysis) that the maximum fin temperature is decreased with the increase of the number of fin array (Fig. 18).

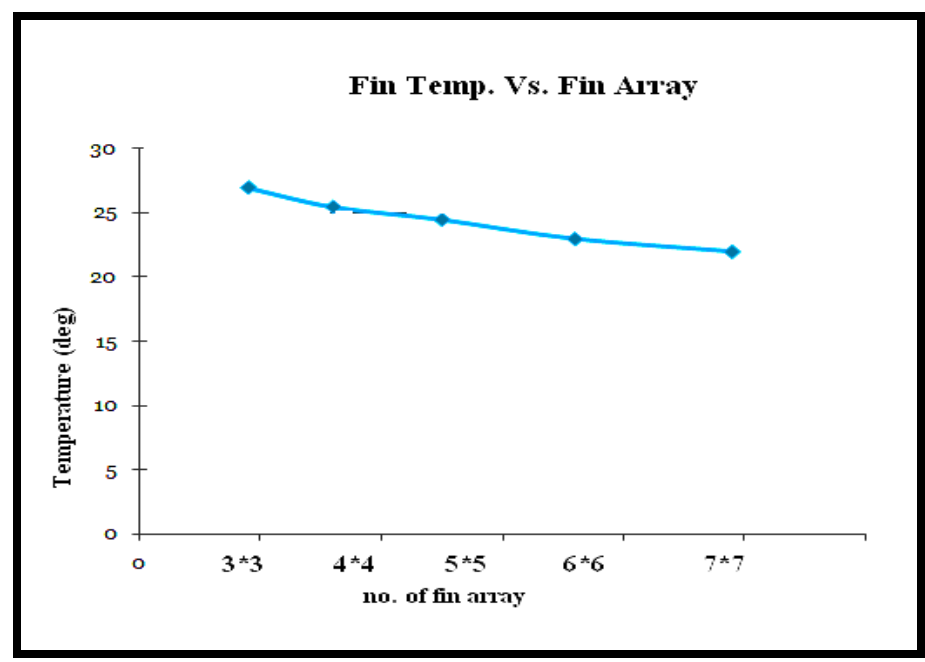

Fig. 18. Fin temperature vs. number of fin array.

\section{Thermal Stress Analysis}

Adhesives are widely used to fabricate CNT on the silicon substrate. Bonded structures are often lighter in weight, lower in cost, and easier to assemble than those mechanical methods. The mismatch in thermal expansion co-efficient (CTEs) will introduce a thermal stress at the bond area when temperature changes. Because of the low modulus of elasticity (E) of adhesives and the thin layer geometry that adhesives are applied in practical usage, the thermal stress caused by adhesives is usually ignorable compare with the stress caused by different CTEs of the bonded parts. But the effects of the adhesives do exist, and can become considerable when other effects have been well controlled. Experiences from optical engineers and opticians show that local fringes can be seen localizing at the bond area due to temperature change. The phenomenon shows that the thermal stress cause by adhesives is able to deflect the mirror surface. This will cause a problem in high requirement system. So it will be helpful to study how the stress develops at the bond area, how it affects the surface, and how it related to the material properties and geometry of adhesives. When two materials with different CTEs are bonded together, for example adhesive and glass, the strain will be forced to be the same (assume 
the bond does not fail). But the different expansion tendency will cause a stress at the interface. Considering the adhesive joint connecting two parts- the adhesive usually has much higher CTE than the connected parts, this makes the adhesive tends to expand more than the jointed parts. The interior part of the adhesive bond is constrained by the adhesive around it, so it can only expand in the direction normal to the bond interface which is not constrained. The difference of expansion between adhesive and jointed parts is small at this area. But the adhesive near the edge is free to bulge laterally, where the adhesive tend to expand more differently to the jointed parts. Thus, the distribution of expansion tendency will introduce a stress distribution. Here for thermal stress analysis all the thermal and physical properties are equal to copper as an assumption [8].

\section{Stress Analysis Result}

Here both rectangular and circular- two dimensional fin array is investigated. Investigation shows that developed von-mises stress and pressure due to temperature difference and fluid flow is less for the circular pin fin array (Fig. 19-22). The maximum pressure in circular fin array is $10.08 \mathrm{MPa}$ and rectangular fin array is $15.14 \mathrm{MPa}$.

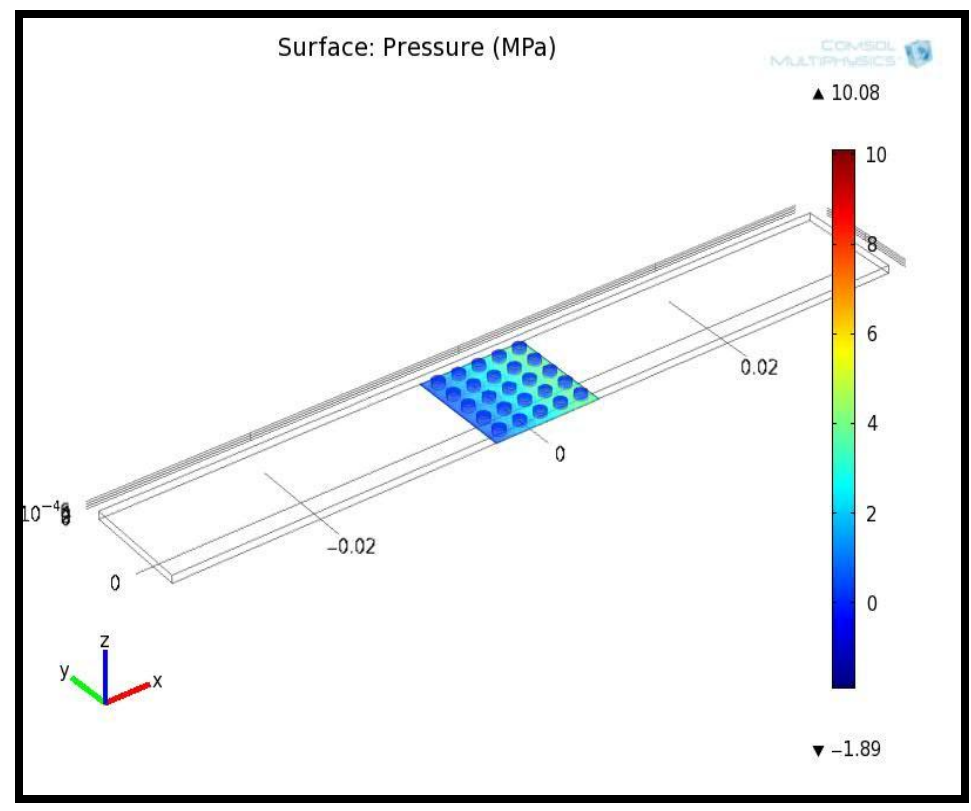

Fig. 19. Pressure distribution in circular fin array.

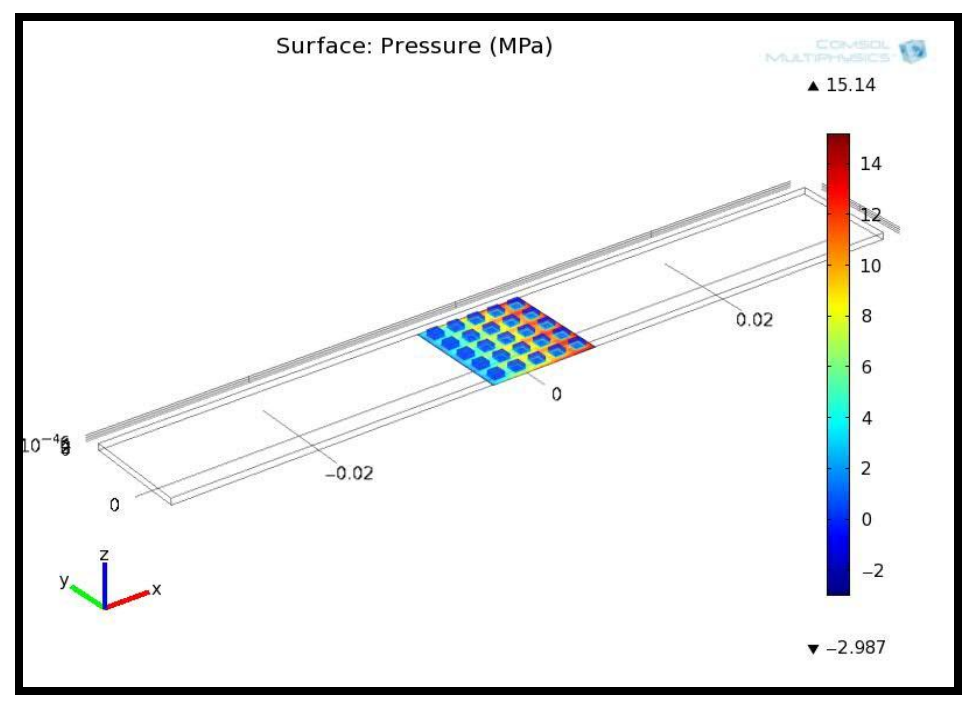

Fig. 20. Pressure distribution in rectangular fin array. 


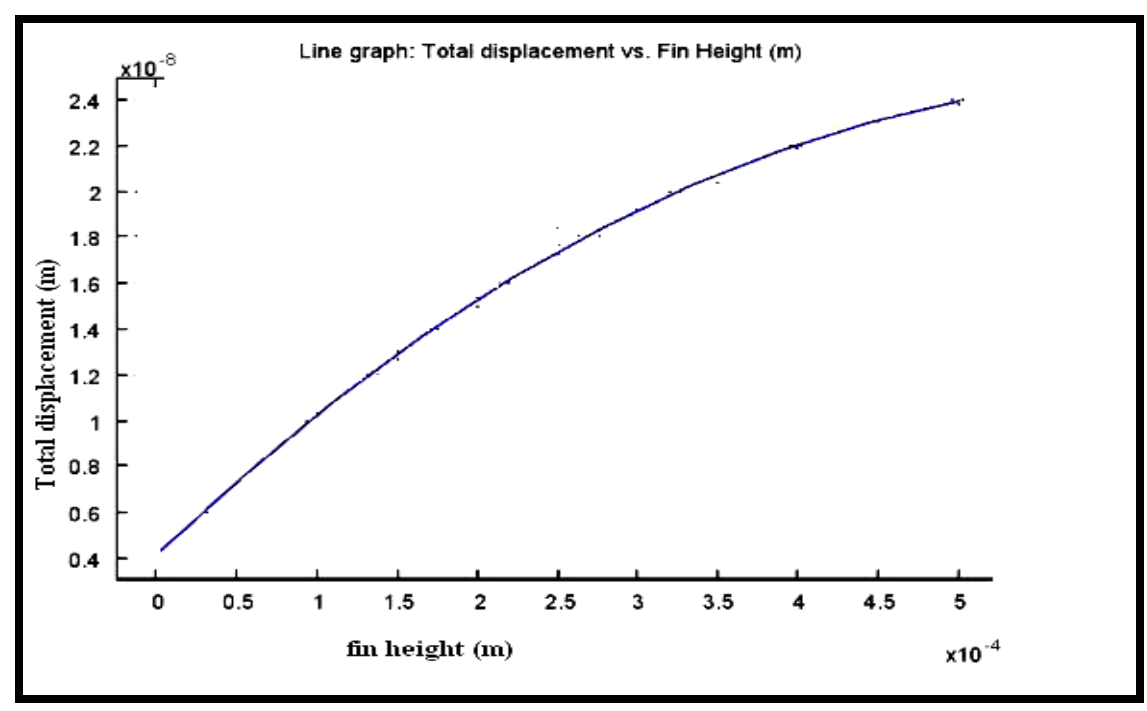

Fig. 21. Total displacement curve along the fin height for circular fin array.

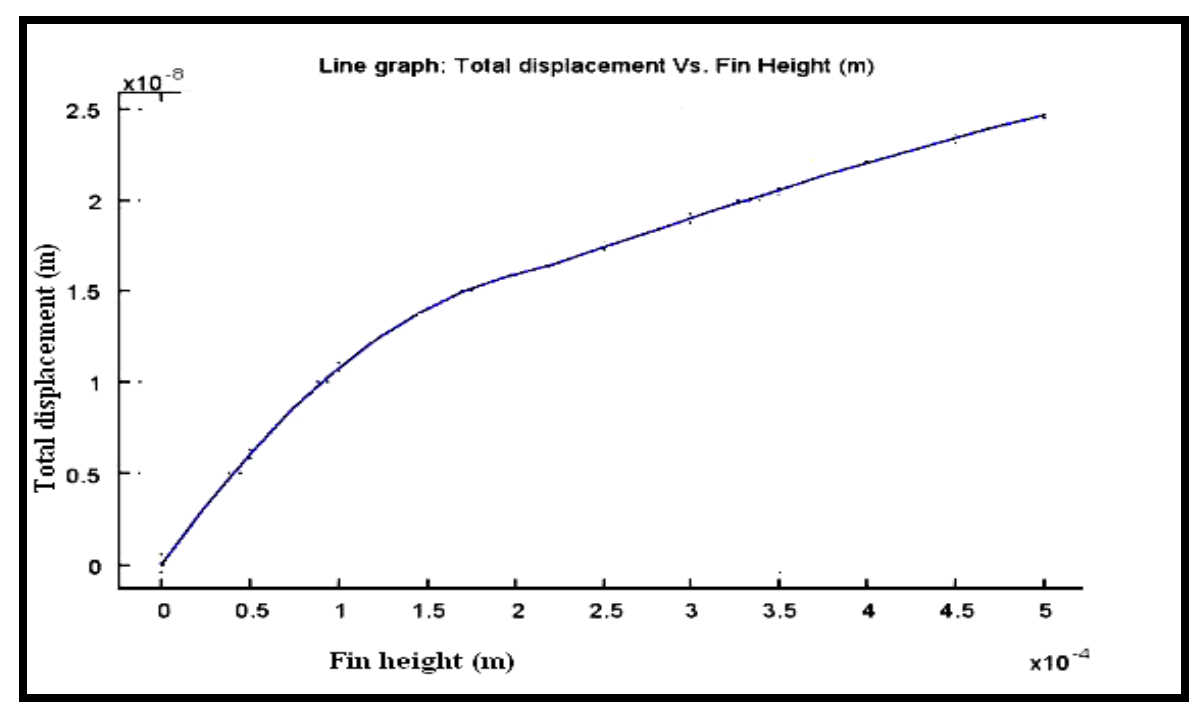

Fig. 22. Total displacement curve along the fin height for rectangular fin array.

\section{Conclusion}

The simulation results in this work indicate that all the heating power is removed by the liquid mass flow. The structures of the micro-fin array, fluid type and the fluid velocity are important factors for heat removal. Heat transfer capability of the micro-fin is dependent very much on the inlet liquid speed. The cooling capability of 2D carbon nanotube (circular) fin array is more efficient than that of $1 \mathrm{D}$ carbon nanotube fin array although 2D fin array causes more pressure drop in the architecture. The 2D CNT fin array can reduce the thermal resistance of the heat sink and the temperature of the CNT fins, and then reduce the temperature of the power chip. Micro-channel heat-sink reliability can be affected by thermal stresses that arise due to temperature gradients between the base and fin along the fin height. Pressure drop in the rectangular fin array is more than the circular one which is not good for the CNT micro-fin array. Though water as a coolant is good among the four fluids (water, ethanol, glycol, $30 \%$ glycol) but due to ionization problem $30 \%$ ethylene glycol may be effective cooling fluid. 


\section{Acknowledgement}

This work was supported by the department of mechanical engineering of Bangladesh University of Engineering \& Technology (BUET).

\section{References}

[1] B. X. Wang and X. F. Peng, "Experimental investigation on liquid forced convection heat transfer through microchannels," Internatinal Journal of Heat and Mass Transfer, vol. 37, pp. 73-82, 1994.

[2] J. Hone, "Carbon Nanotubes: Thermal properties," Dekker Encyclopedia of Nanoscience and Nanotechnology, pp. 603-610.

[3] J. Liu, Y. Fu, and T. Wang, "Recent progress of carbon nanotubes as cooling fins in electronic packaging", International Conference on Electronic Packaging Technology \& High Density Packaging, 2008.

[4] J. Xu and T. S. Fisher, "Enhancement of thermal interface materials with carbon nanotube arrays," International Journal of Heat and Mass Transfer, vol. 49, pp. 1658-1666, 2006.

[5] K. Kordas, G. Toth, P. Moilanen, M. Kumpumaki, and A. Uusimaki, "Chip cooling with integreted carbon nanotube microfin architectures", Applied Physics Letters, vol. 90, 123105(2007).

[6] S. Khandlikar, S. Garimella, S. Colin, and M. R. King, Heat Transfer and Fluid Flow in Minichannels and Microchannels, 1st ed. India: 2006.

[7] S. Wang, Y. Zhang, Y. Fu, X. Wang, and Z. Cheng, "A study of heat transfer characterstics of the micro-channel heatsink," International Conference on Electronic Packaging Technology \& High Density Packaging, 2009, pp. 255-259.

[8] T. Tong, Y. Zhao, L. Delzeit, A. Kashani, M. Meyyappan, and A. Majumdar, "Dense vertically aligned multiwalled carbon nanotube arrays as thermal interface materials," IEEE Transactions on Components and Packaging Technologies, vol. 30, pp. 92-100, 2007.

[9] Z. Mo, Ra. Morjan, J. Anderson, E. E. B. Campbell, and J. Liu, "Integrated nanotube microcooler for microelectronics applications," Electronic Components and Technology Conference, 2005, pp. 51-54.

[10] Z. Xiaolong, Y. Fan, J. Liu, Y. Zhang, T. Wang, and Z. Cheng, "A study of CFD simulation for on-chip cooling with 2D CNT micro-fin array," International Symposium on High Density Packaging and Microsystem Integration, China, 2007, pp. 442-447. 